International Journal of Biomathematics

Vol. 7, No. 6 (2014) 1499001 (7 pages)

(C) World Scientific Publishing Company

DOI: $10.1142 / \mathrm{S} 1793524514990010$

\title{
Author index (Volume 7)
}

Abukwaik, R. M., see

Elaiw, A. M.

Adlakha, N., see Jha, B. K.

Adlakha, N., see Mathur, R.

Aijaz, M., Khanday, M. A. \& Rafiq, A., Variational finite element approach to study the thermal stress in multi-layered human head

Akbar, N. S. \& Butt, A. W., Heat transfer analysis of viscoelastic fluid flow due to metachronal wave of cilia

Akbar, N. S. \& Nadeem, S., Blood flow analysis in tapered stenosed arteries with pseudoplastic characteristics

Akbar, N. S., see Riaz, A.

Akbar, N. S., Blood flow of Carreau fluid in a tapered artery with mixed convection

Akbar, N. S., Heat and mass transfer effects on Carreau fluid model for blood flow through a tapered artery with a stenosis

Alotaibi, N., see Yasmin, $\mathrm{H}$.

Alsaadi, F., see Hayat, T.

Alsaedi, A., see Awais, M.
1 (2014) 1450004

5 (2014) 1450058

5 (2014) 1450057

5 (2014) 1450055

3 (2014) 1450031

2 (2014) 1450012

6 (2014) 1450073

6 (2014) 1450066

6 (2014) 1450065

5 (2014) 1450049

6 (2014) 1450068

3 (2014) 1450023
Alsaedi, A., see Hina, S.

Alsaedi, A., see Saleem, N.

Alzahrani, E. O., see

Elaiw, A. M.

Arafa, A. A. M., Rida, S. Z. \& Khalil, M., A fractional-order model of HIV infection:

Numerical solution and comparisons with data of patients

Asif, W., Qureshi, H. K., Iqbal, A. \& Rajarajan, M., On the complexity of average path length for biological networks and patterns

4 (2014) 1450038

Athithan, S. \& Ghosh, M., Analysis of a sex-structured $H I V / A I D S$ model with the effect of screening of infectives

5 (2014) 1450054

Awais, M., Farooq, S., Yasmin, H., Hayat, T. \& Alsaedi, A.,

Convective heat transfer analysis for MHD peristaltic flow in an asymmetric channel

3 (2014) 1450023

Bai, Y.-P., see Wang, X.-Y.

4 (2014) 1450043

Balachandran, K., see Shangerganesh, L.

6 (2014) 1450062

Balasubramaniam, P., see Prakash, M.

6 (2014) 1450070

Barani Balan, N., see

Shangerganesh, L.

Bekir, A., see Jabbari, A.

6 (2014) 1450062

3 (2014) 1450025 
Belmonte-Beitia, J., On

the existence of

traveling wave

solutions and upper

and lower bounds for

some Fisher-

Kolmogorov type equations

5 (2014) 1450050

Bhattacharjee, A., Das,

M. K. \& Ghosh, S.,

Synchronization in a

ring of unidirectionally

coupled

Fitzhugh-Nagumo

neurons

1 (2014) 1450009

Bhunu, C. P., see

Mushayabasa, S.

Bougoffa, L., On the solutions of an oxygen absorption model

Butt, A. W., see Akbar, N. S.

Cheke, R. A., see Wang, Q.

Chen, F., see $\mathrm{Yu}, \mathrm{S}$.

Chen, L., see Tian, Y.

Das, M. K., see

Bhattacharjee, A.

Ding, L., see Zhao, K.

Dong, Q. \& Ma, W., Qualitative analysis of a chemostat model with inhibitory exponential substrate uptake and a time delay

Duan, J.-S., see Rach, R.

Elaiw, A. M., Abukwaik, R. M. \& Alzahrani, E. O., Global properties of a cell mediated immunity in HIV infection model with two classes of target cells and distributed delays

5 (2014) 1450055

5 (2014) 1450049

, see Riaz, A.

Elsayed, E. M., On the solutions and periodic nature of some systems of difference equations

Er-Dong, H. \& Peng, G., Dynamical behaviors

1 (2014) 1450006

6 (2014) 1450061

6 (2014) 1450066

4 (2014) 1450040

3 (2014) 1450028

2 (2014) 1450018

1 (2014) 1450009

1 (2014) 1450003

4 (2014) 1450045

2 (2014) 1450020 of a diffusive

predator-prey system with Beddington-

DeAngelis functional response

3 (2014) 1450033

Fan, Y.-H., see Wang, L.-L.

Farooq, S., see Awais, M.

1 (2014) 1450007

3 (2014) 1450023

Fattorini, L. \&

Lacitignola, D.,

Functionality indexes assessed through a simple model of muscle activation, fatigue and recovery

2 (2014) 1450022

Feng, Y. \& Luo, L., Using long-range contact number information for protein secondary structure prediction

Fu, L., Tang, S., Liu, Y., Sharma, R. P., Zhang, H., Lei, Y., Wang, H. \& Song, X., Developing, testing and application of rodent population dynamics and capture models based on an adjusted Leslie matrix-based population approach 3 (2014) 1450024 5 (2014) 1450058 Gao, H., see Yasmin, H. Ghosh, M., see Athithan, S.

5 (2014) 1450054

Ghosh, S., see

Bhattacharjee, A.

Götz, T., see Wijaya, K. P.

1 (2014) 1450009

5 (2014) 1450056

Goyal, A., see Shukla, J. B.

1 (2014) 1450008

Guin, L. N. \& Mandal, P. K., Spatial pattern in a diffusive predatorprey model with sigmoid ratio-dependent functional response

5 (2014) 1450047
Hayat, T., Iqbal, M., Yasmin, H. \& Alsaadi, F., Hall effects on peristaltic flow of 
couple stress fluid in an inclined

asymmetric channel

Hayat, T., see Awais, M.

Hayat, T., see Hina, S.

Hayat, T., see Saleem, N.

Hayat, T., see Yasmin, $\mathrm{H}$.

He, Z.-R. \& Liu, R., Theory of optimal harvesting for a nonlinear size-structured population in periodic environments

5 (2014) 1450057

3 (2014) 1450023

2 (2014) 1450015

1 (2014) 1450010

5 (2014) 1450058

4 (2014) 1450046

Heris, J. M., see Jabbari, A.

Hina, S., Hayat, T., Mustafa, M. \&

Alsaedi, A., Peristaltic transport of pseudoplastic fluid in a curved channel with wall properties and slip conditions

Hu, G., Li, X. \& Lu, S., Stationary patterns for a Leslie-Gower type three species model with diffusion

Huang, M. \& Song, X., Modeling and qualitative analysis of diabetes therapies with state feedback control

Huang, T., see Zhang, W.

Iqbal, A., see Asif, W. Iqbal, M., see Hayat, T. Jabbari, A., Heris, J. M., Kheiri, H. \& Bekir, A., $A$ generalization of $\left(G^{\prime} / G\right)$-expansion method and its application to nonlinear

reaction-diffusion equations arising in mathematical biology Javidi, M. \& Nyamoradi, N., A fractional-order toxin producing phytoplankton and zooplankton system

4 (2014) 1450035

2 (2014) 1450016

4 (2014) 1450038

5 (2014) 1450057

3 (2014) 1450025

4 (2014) 1450039
Jha, B. K., Adlakha, N.

\& Mehta, M. N.,

Two-dimensional finite element model to study calcium distribution in astrocytes in presence of excess buffer

3 (2014) 1450031

Jiang, J. \& Niu, L., The dynamical behavior on the carrying simplex of a three-dimensional competitive system: II. Hyperbolic structure saturation

Jiang, J. \& Niu, L., The theorem of the carrying simplex for competitive system defined on the $n$-rectangle and its application to a three-dimensional system

Jiang, W., Li, X. \& Zou, $\mathrm{X}$., On a reactiondiffusion model for sterile insect release method on a bounded domain

Kamal, Md. S. \& Khan, M. I., ChapmanKolmogorov equations for global PPIs with Discriminant-EM

Kameswaran, P. K., see Shaw, S.
Katiyar, V. K., see Saini, A.

Khalil, M., see Arafa, A. A. M.

Khan, M. I., see Kamal, Md. S.

Khanday, M. A., see Aijaz, M.

Kheiri, H., see Jabbari, A.

Kordrostami, S., Efficiency decomposition in parallel production systems with shared sources on interval data: An illustration of Iranian Banks

Lacitignola, D., see Fattorini, L.
3 (2014) 1450030

5 (2014) 1450059

1 (2014) 1450002

6 (2014) 1450063

5 (2014) 1450053

1 (2014) 1450005

2 (2014) 1450021

4 (2014) 1450036

5 (2014) 1450053

6 (2014) 1450073

3 (2014) 1450025

2 (2014) 1450022 
Lakshminarayana, P., see Vajravelu, K.

6 (2014) 1450064

Laxmi, D., see Rathod, V. P.

Lei, Y., see Fu, L.

Li, A., see Wan, X.-K.

Li, C., see Zhang, W.

Li, J.-W., see Wang, F.

Li, X., see Hu, G.

Li, X., see Jiang, W.

Li, Z., see Wang, Q.

Liao, G.-P., see Wang, F.

Lin, Z., see Tian, C.

Ling, Z., see Tian, C.

Liu, J., Bifurcation analysis in a stage-structured predator-prey model with maturation delay

Liu, R., see He, Z.-R.

Liu, Y., see Fu, L.

Liu, Z., see Wang, Q.

Liu, Z.-Q., see Wang, F.

$\mathrm{Lu}, \mathrm{S}$, see $\mathrm{Hu}, \mathrm{G}$.

Luo, L., see Feng, Y.

Luo, Y., see Zhu, H.

Ma, W., see Dong, Q.

Makinde, O. D., see

Okosun, K. O.

Mandal, P. K., see Guin, L. N.

Mathur, R. \& Adlakha,

N., Binary

sequences-based

approach for

construction of evolutionary network

Mehta, M. N., see Jha, B. K.

Misra, A. K., see Shukla, J. B.

Modnak, C., Wang, J. \& Mukandavire, Z., Simulating optimal vaccination times during cholera outbreaks

Mohamed, K. G., see Mohammadein, S. A.

Mohammadein, S. A. \& Mohamed, K. G., Growth of gas bubbles in the biotissues with convective acceleration
6 (2014) 1450060

3 (2014) 1450024

4 (2014) 1450044

2 (2014) 1450016

5 (2014) 1450048

6 (2014) 1450069

3 (2014) 1450030

4 (2014) 1450040

5 (2014) 1450048

2 (2014) 1450013

2 (2014) 1450013

4 (2014) 1450042

4 (2014) 1450046

3 (2014) 1450024

4 (2014) 1450040

5 (2014) 1450048

6 (2014) 1450069

5 (2014) 1450052

3 (2014) 1450034

4 (2014) 1450045

2 (2014) 1450019

5 (2014) 1450047

2 (2014) 1450012

3 (2014) 1450031

1 (2014) 1450008

2 (2014) 1450014

6 (2014) 1450072

6 (2014) 1450072
Montaseri, G. \&

Yazdanpanah, M. J., Desynchronization of two coupled limit-cycle oscillators using an astrocyte-inspired controller

1 (2014) 1450001

Mukandavire, Z., see Modnak, C.

Mushayabasa, S. \& Bhunu, C. P., Hepatitis $C$ virus and intravenous drug misuse: A modeling approach

1 (2014) 1450006

Mustafa, M., see Hina, S.

2 (2014) 1450015

Nadeem, S., see Akbar, N. S.

6 (2014) 1450065

5 (2014) 1450049

Nadeem, S., see Riaz, A.

Narayana, M., see Shaw, S.

1 (2014) 1450005

1 (2014) 1450002

6 (2014) 1450063

Niu, L., see Jiang, J.

Nyamoradi, N., see

Javidi, M.

4 (2014) 1450039

Okosun, K. O. \&

Makinde, O. D.,

Optimal control

analysis of hepatitis $C$

virus with acute and

chronic stages in the

presence of treatment

and infected

immigrants

Peng, G., see Er-Dong, H.

2 (2014) 1450019

3 (2014) 1450033

Prakash, M. \&

Balasubramaniam, P., Mathematical analysis of a model for thymus infection with discrete and distributed delays

6 (2014) 1450070

2 (2014) 1450021

Pratibha, see Saini, A

Qureshi, H. K., see Asif, W.

4 (2014) 1450038

Rach, R., Wazwaz, A.-M.

\& Duan, J.-S., A

reliable analysis of oxygen diffusion in a spherical cell with nonlinear oxygen uptake kinetics

2 (2014) 1450020

Rafiq, A., see Aijaz, M.

6 (2014) 1450073 
Rajarajan, M., see Asif, W.

4 (2014) 1450038

Rathod, V. P. \& Laxmi, D., Effects of heat transfer on the peristaltic $M H D$ flow of a Bingham fluid through a porous medium in a channel

Riaz, A., Nadeem, S., Ellahi, R. \& Akbar, N. S., Series solution of unsteady peristaltic flow of a Carreau fluid in small intestines

Rida, S. Z., see Arafa, A. A. M.

Saini, A., Katiyar, V. K. \& Pratibha, Effects of first-order chemical reactions on the dispersion coefficient associated with laminar flow through the lungs

Saleem, N., Hayat, T. \& Alsaedi, A., A

hydromagnetic mathematical model for blood flow of Carreau fluid

Shangerganesh, L., Barani Balan, N. \& Balachandran, K., Weak-renormalized solutions for three species competition model in ecology

Sharma, R. P., see Fu, L.

Shaw, S., Kameswaran, P. K., Narayana, M. \& Sibanda, P., Bioconvection in a non-Darcy porous medium saturated with a nanofluid and oxytactic micro-organisms

Shukla, J. B., Goyal, A., Tiwari, P. K. \& Misra, A. K., Modeling the role of dissolved oxygen-dependent bacteria on

6 (2014) 1450060

4 (2014) 1450036

2 (2014) 1450021

1 (2014) 1450010

6 (2014) 1450062

3 (2014) 1450024 biodegradation of

organic pollutants

1 (2014) 1450008

Sibanda, P., see Shaw, S.

Soewono, E., see Wijaya,

K. P.

1 (2014) 1450005

Song, X., see Fu, L.

5 (2014) 1450056

3 (2014) 1450024

Song, X., see Huang, M.

Sreenadh, S., see

Vajravelu, K.

4 (2014) 1450035

Sucharitha, G., see

Vajravelu, K.

6 (2014) 1450064

6 (2014) 1450064

Sun, J., see Zhang, J.

Sun, K., see Tian, Y.

Tang, S., see Fu, L.

Tang, X., see Yang, $\mathrm{P}$.

Tian, C., Ling, Z. \& Lin, Z., Spatial patterns created by cross-diffusion for a three-species food chain model

3 (2014) 1450032

2 (2014) 1450018

3 (2014) 1450024

2 (2014) 1450017

Tian, Y., Sun, K. \& Chen, L., Geometric approach to the stability analysis of the periodic solution in a semi-continuous dynamic system

2 (2014) 1450018

Tiwari, P. K., see Shukla, J. B.

1 (2014) 1450008

Vajravelu, K., Sreenadh, S., Sucharitha, G. \& Lakshminarayana, P., Peristaltic transport of a conducting Jeffrey fluid in an inclined asymmetric channel

Wan, X.-K., Yan, K.-H., Li, A. \& Wu, M.-H., Improved modified moving average analysis of $T$-wave alternans using least squares-based curve fitting method

4 (2014) 1450044

Wang, C.-Y., see Zhang, Y.-J.

2 (2014) 1450011

Wang, F., Zou, R.-B. Liao, G.-P., Li, J.-W. \& Liu, Z.-Q., Local multifractal detrended fluctuation analysis for tea breeds identification

Wang, F., see Wang, W.
4 (2014) 1450037 
Wang, H., see Fu, L.

Wang, J., see Modnak, C.

Wang, K. \& Zhu, Y.,

Dynamics of $a$

stochastic

predator-prey model

with mutual

interference

Wang, K. \& Zhu, Y.,

Global attractivity of

positive periodic

solution for a

predator-prey model

with modified

Leslie-Gower

Holling-type II

schemes and a

deviating argument

Wang, L.-L., Zhou, B.-B.

\& Fan, Y.-H., Hopf

bifurcation analysis for

a delayed Leslie-

Gower predator-prey

system with diffusion

effects

Wang, Q., Liu, Z., Li, Z.

\& Cheke, R. A.,

Existence and global

asymptotic stability of

positive almost

periodic solutions of a

two-species competitive

system

Wang, W., Xiong, Z. \&

Wang, F., Dynamic

analysis of a

stage-structured

predator-prey system

with disturbed time

delay and birth pulse

Wang, X., see Zhu, H.

Wang, X.-Y., Bai, Y.-P.,

Yang, J.-Y. \& Zhang,

F.-Q., Global stability

of an epidemic model

for $H I V-T B$

co-infection with

infection-age

Wazwaz, A.-M., see

Rach, R.

Wen, X., see Yin, H.

Wijaya, K. P., Götz, T.

\& Soewono, E., An

optimal control model
3 (2014) 1450024

2 (2014) 1450014

3 (2014) 1450026

6 (2014) 1450071

1 (2014) 1450007

4 (2014) 1450040

3 (2014) 1450034

4 (2014) 1450043

2 (2014) 1450020

5 (2014) 1450051
4 (2014) 1450037

of mosquito reduction

management in a

dengue endemic

region

5 (2014) 1450056

Wu, M.-H., see Wan, X.-K.

4 (2014) 1450044

5 (2014) 1450051

4 (2014) 1450037

Xiong, Z., see Wang, W.

$\mathrm{Xu}$, J., Xu, W. \& Zhou, Y., Analysis of a delayed epidemic model with

non-monotonic incidence rate and vertical transmission

$\mathrm{Xu}, \mathrm{W}$., see $\mathrm{Xu}, \mathrm{J}$.

Yan, K.-H., see Wan, X.-K.

Yang, F., see Zhao, K.

Yang, J.-Y., see Wang, X.-Y.

Yang, P. \& Tang, X., Exponential synchronization for neural networks with mixed time-varying delays via periodically intermittent control

Yang, W., Permanence of a diffusive

Leslie-Gower predator-prey model incorporating a prey refuge

Yasmin, H., Hayat, T., Alotaibi, N. \& Gao, H., Convective heat and mass transfer analysis on peristaltic flow of Williamson fluid with Hall effects and Joule heating

Yasmin, H., see Awais, M.

Yasmin, H., see Hayat, $\mathrm{T}$.

Yazdanpanah, M. J., see Montaseri, G.

Yin, H., Xiao, X., Wen, X. \& Zhou, T., Mathematical analysis on a multidimensional model of morphogen transport with receptor synthesis
4 (2014) 1450041

4 (2014) 1450041

4 (2014) 1450044

1 (2014) 1450003

4 (2014) 1450043

2 (2014) 1450017

3 (2014) 1450027

5 (2014) 1450058

3 (2014) 1450023

5 (2014) 1450057

1 (2014) 1450001
5 (2014) 1450051 
Yu, S. \& Chen, F., Almost periodic solution of a modified Leslie-Gower predator-prey model with Holling-type II schemes and mutual interference

Zhang, F.-Q., see Wang, X.-Y.

Zhang, H., see Fu, L.

Zhang, J. \& Sun, J., A delayed SEIRS epidemic model with impulsive vaccination and nonlinear incidence rate

Zhang, T., Almost periodic oscillations in a generalized Mackey-Glass model of respiratory dynamics with several delays

Zhang, W., Li, C. \& Huang, T., Global robust stability of complex-valued recurrent neural networks with time-delays and uncertainties
3 (2014) 1450028

4 (2014) 1450043

3 (2014) 1450024

3 (2014) 1450032

3 (2014) 1450029

2 (2014) 1450016
Zhang, Y.-J. \& Wang, C.-Y., Stability analysis of $n$-species Lotka-Volterra almost periodic competition models with grazing rates and diffusions

2 (2014) 1450011

Zhao, K., Ding, L. \& Yang, F., Existence of multiple periodic solutions to LotkaVolterra network-like food-chain system with delays and impulses on time scales

1 (2014) 1450003

Zhou, B.-B., see Wang, L.-L.

1 (2014) 1450007

5 (2014) 1450051

4 (2014) 1450041

Zhou, T., see Yin, H

Zhou, Y., see Xu, J.

Zhu, H., Luo, Y. \& Wang, X., Traveling wavefronts of a nonlinear reactiondiffusion model of tumor growth under the acid environment $\mathbf{3}$ (2014) 1450034 Zhu, Y., see Wang, K. 3 (2014) 1450026 Zhu, Y., see Wang, K. 6 (2014) 1450071 Zou, R.-B., see Wang, F. 5 (2014) 1450048 Zou, X., see Jiang, W. 3 (2014) 1450030 